Carlo Victorio L. Garcia, MD Arsenio Claro A. Cabungcal, MD

Alfredo Quintin Y. Pontejos, Jr., MD

Department of Otorhinolaryngology Philippine General Hospital

University of the Philippines Manila

\section{Prognostic Value of Thyroidectomy and Tracheostomy in Anaplastic Thyroid Carcinoma}

\begin{abstract}
Objective: To determine the prognostic value of surgical interventions done among patients with anaplastic thyroid carcinoma (ATC)

Methods: A five-year retrospective chart review of 25 patients was done and baseline characteristics determined. Patients discharged alive as of the time of last chart entry were followed up by phone interview or personal visit. Overall survival was the main outcome measure which was plotted as Kaplan-Meier estimates and compared via log-rank test. The incidence of complications surrounding tracheostomy and thyroidectomy were also noted.
\end{abstract}

\section{Methods:}

\section{Design: Ambispective Cohort Study \\ Setting: $\quad$ Tertiary National University Hospital}

Participants: $\quad$ All private and public (charity) patients seen at the wards or clinics diagnosed with ATC via fine needle cytology or tissue histopathology.

The authors declared that this represents original materia that is not being considered for publication or has not been published or accepted for publication elsewhere in full or in part, in print or electronic media; that the requirements for authorship have been met by all the authors, and that each author believes that the manuscript represents honest work.

Disclosures: The authors signed a disclosure that there are no financial or other (including personal) relationships, intellectual passion, political or religious beliefs, and institutional affiliations that might lead to a conflict of interest.

Presented at the 4th European Otolaryngology-ENT Surgery Conference, August 15-17, 2019, Rome, Italy.

Presented at the Philippine Society of Otolaryngology Head and Neck Surgery Descriptive Research Contest. December 6 2019. Palawan Ballroom, Edsa Shangri La Hotel, Mandaluyong City.

\section{(ब) $(\Theta \Theta$}

Results: All patients presented with either stage IV-B or stage IV-C disease. A significant difference in survival curves was noted when comparing between the two stages $(p<.05)$. Subgroup analysis per stage revealed no significant difference in overall survival when comparing patients who did not undergo surgery, those who underwent tracheostomy or those who underwent thyroidectomy for both IV-B ( $p=.244)$ or IV-C $(p=.165)$ disease. The incidence of complications for tracheostomy was $60 \%$, the most common being mucus plugging. For thyroidectomy, the incidence of complications was $80 \%$ with hypocalcemia being the most common.

Conclusion: The current available data fails to demonstrate any significant survival advantage of tracheostomy or thyroidectomy when performed among similarly staged patients.

Keywords: anaplastic thyroid cancer; thyroidectomy; tracheostomy; survival 
Of all the known malignancies, anaplastic thyroid carcinoma (ATC) carries one of the worst prognosis with a 5 -year survival rate of only $5.6-11.4 \%$ and a median life expectancy of only $2-12$ months. ${ }^{1-5}$ The incidence of ATC is pegged at 1 to 2 cases per million people.? Despite being the least common thyroid malignancy, it accounts for roughly $50 \%$ of all thyroid cancer deaths. ${ }^{6}$ All anaplastic cancers are automatically stage IV upon diagnosis: IV-A is disease limited to the thyroid, IV-B is tumor invading through thyroid capsule and/or regional metastasis and IV-C involves distant metastasis. At least $90 \%$ of patients with ATC are elderly (>60 years) at presentation. ${ }^{7,8}$ Because of this, and the advanced disease at presentation, surgical efforts at complete resection remain controversial as they are associated with significant morbidity and reduced quality of life. In our institution, most of these patients are treated with palliative rather than curative intent with most of the procedures geared towards relief of airway obstruction. Recently however, cohort studies with sizeable patient pools in Italy and Korea have shown significant survival benefit for curative resection when performed among appropriately selected patients..$^{910}$

A search of local literature via MEDLINE (PubMed) and HERDIN only yielded two descriptive studies on ATC among Filipinos. Sunga et al. performed a retrospective study of 17 patients from 1973-1994 and compared the outcomes of thyroidectomy and radiotherapy in resectable ATC versus tracheostomy and radiotherapy in unresectable ATC. ${ }^{11}$ Although they determined that combined thyroidectomy and radiotherapy resulted in a longer mean survival, this was not analyzed with respect to staging. ${ }^{11} \mathrm{~A}$ more recent study by Lo et al. detailed the symptomatology, demographics and clinical course of these patients. However, it did not describe the survival benefit of surgical interventions done on these patients nor did it mention the complications or morbidities surrounding these procedures. ${ }^{12}$

Considering recent evidence that refocuses our attention on surgery as the primary modality for curative efforts, we aim to validate if this was applicable at the local setting as well. This study aims to determine the prognostic value of surgical interventions done among patients with anaplastic thyroid carcinoma (ATC) in our institution.

\section{METHODS}

With Institutional Review Board approval, this ambispective cohort study was conducted among all patients with cytologically or histopathologically confirmed anaplastic thyroid carcinoma admitted at the in-patient ward or seen at the out-patient clinic of the Department of Otorhinolaryngology of the Philippine General Hospital from January 2013 to January 2018. Securing informed consent was waived for patients who expired while admitted and whose death certificate was already available in the records. For patients who were discharged alive but died sometime after, informed consent was obtained from the nearest kin.

All the inpatient and outpatient charts of patients with either initial cytopathologic diagnosis of ATC via fine needle biopsy and/ or final histopathologic confirmation of ATC within the study period were reviewed. Excluded were patients with an initial diagnosis of ATC refuted by subsequent histopathological examination.

Patient demographics and baseline characteristics were obtained from the selected charts. Patients were further classified into one of 3 groups: 1) no surgical intervention, 2) tracheostomy with or without debulking, 3) total thyroidectomy/wide excision of tumor with or without neck dissection. The rates of intraoperative and postoperative complications were determined for each surgical intervention done.

Disease-specific mortality was the primary endpoint. If the chart reflected that the patient was discharged alive as of the time of last admission/consult, the current status was verified by a recorded phone call to the contact person identified in the records or a personal visit at the listed address. Information gathered was limited to the date of death (if applicable) and cause of death (if applicable). If the patient or contact person was not reachable through listed channels (through phone or personal visit at listed address), they were censored in the final analysis.

Kaplan-Meier disease-specific survival curves were generated comparing each stage followed by subgroup analysis per stage of the different interventions the patients received. Survival estimates were compared via log-rank test using SPSS 17 (SPSS Inc., Chicago IL).

\section{RESULTS}

A total of 25 patients had charts available for review for the time period covered. There was a 3:1 female to male preponderance and a median age of presentation of 59 years old (range of 44 to 87 years old). The baseline characteristics and the surgical interventions performed for each patient are summarized in Table 1.

The primary outcome was determined in only 13 of the 25 patients. The rest were censored as of the time of last chart entry or clinic visit.

The top causes of death among stage IV-B patients were sepsis/ respiratory failure from pneumonia, arrhythmia from myocardial infarction and upper airway obstruction. The top causes of death among stage IV-C patients were sepsis from pneumonia and multiorgan dysfunction from tumor metastasis. The mean overall survival of stage IV-B patients was 5 weeks while that of IV-C patients was only 2 weeks. Figure 1 shows the Kaplan-Meier curves grouped according to stage. As expected, log-rank test showed that the survival probability of stage IV-B patients was significantly different from IV-C patients ( $p$ value $=.01$ ).

Figures 2 and 3 show a subgroup analysis per stage and compared 


\section{ORIGINAL ARTICLES}

the various surgical interventions done on these patients. Comparing same stage patients, the data shows that neither tracheostomy nor thyroidectomy significantly altered the survival of patients compared to those who did not undergo any surgical intervention ( $p$ value $=.21$ for IV-B, $\mathrm{p}$ value $=.17$ for IVC).

Thyroidectomies were associated with postoperative hypocalcemia $40 \%$ of the time. Tracheostomies were associated with complications $62 \%$ of the time and the most common was mucus plugging. Other tracheostomy complications included tube infiltration by tumor, surgical site infections and bleeding per stoma.

\section{DISCUSSION}

This study corroborates the previous findings of Lo et al. that demonstrated similar symptomatology among their cohort of patients. ${ }^{12}$ Aside from a rapidly enlarging neck mass, dysphagia was the next most common complaint, occurring in $72 \%$ of patients.

Table 1. Clinical characteristics of 25 ATC patients and enumeration of surgical interventions performed per stage

\begin{tabular}{|c|c|c|}
\hline Factors & \multicolumn{2}{|c|}{ Cases $(n=25)$} \\
\hline \multicolumn{3}{|l|}{ Gender } \\
\hline Female & \multicolumn{2}{|c|}{19} \\
\hline \multicolumn{3}{|l|}{ Age } \\
\hline$<60$ years & \multicolumn{2}{|c|}{13} \\
\hline$\geq 60$ years & \multicolumn{2}{|c|}{12} \\
\hline \multicolumn{3}{|l|}{ Number of symptomatic months prior to diagnosis } \\
\hline$<1$ month & \multicolumn{2}{|c|}{2} \\
\hline $1-3$ months & \multicolumn{2}{|c|}{13} \\
\hline$>3$ months & \multicolumn{2}{|c|}{10} \\
\hline \multicolumn{3}{|l|}{ Nodal metastasis } \\
\hline No & \multicolumn{2}{|c|}{10} \\
\hline Yes & \multicolumn{2}{|c|}{15} \\
\hline \multicolumn{3}{|l|}{ Resectability of primary tumor } \\
\hline Yes & \multicolumn{2}{|c|}{11} \\
\hline No & \multicolumn{2}{|c|}{9} \\
\hline Equivocal & \multicolumn{2}{|c|}{5} \\
\hline \multicolumn{3}{|l|}{ Stage } \\
\hline IV-B & \multicolumn{2}{|c|}{15} \\
\hline IV-C & \multicolumn{2}{|c|}{10} \\
\hline & IV-B & IV-C \\
\hline \multicolumn{3}{|l|}{ Surgical intervention } \\
\hline None & 3 & 2 \\
\hline Tracheostomy (with or without debulking) & 8 & 7 \\
\hline Thyroidectomy (with or without neck dissection) & 4 & 1 \\
\hline
\end{tabular}

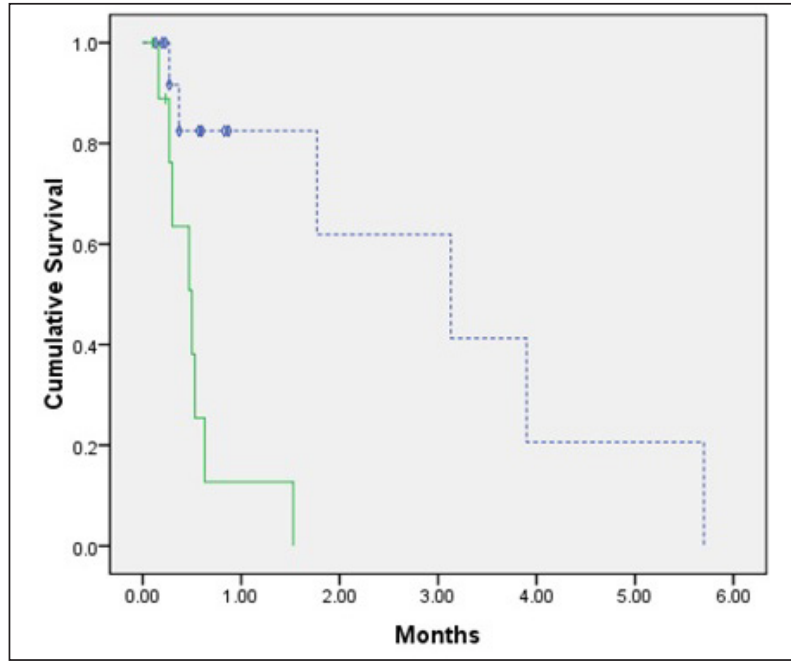

Figure 1. Kaplan-Meier curves showing the effect of staging on overall survival

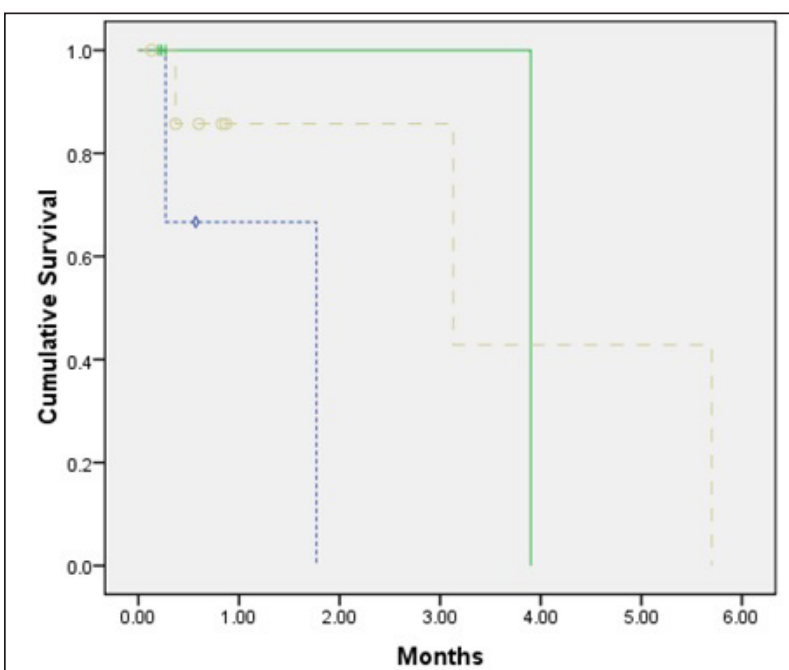

Figure 2. Kaplan-Meier curves showing effect of surgical interventions on overal survival of stage IVB patients

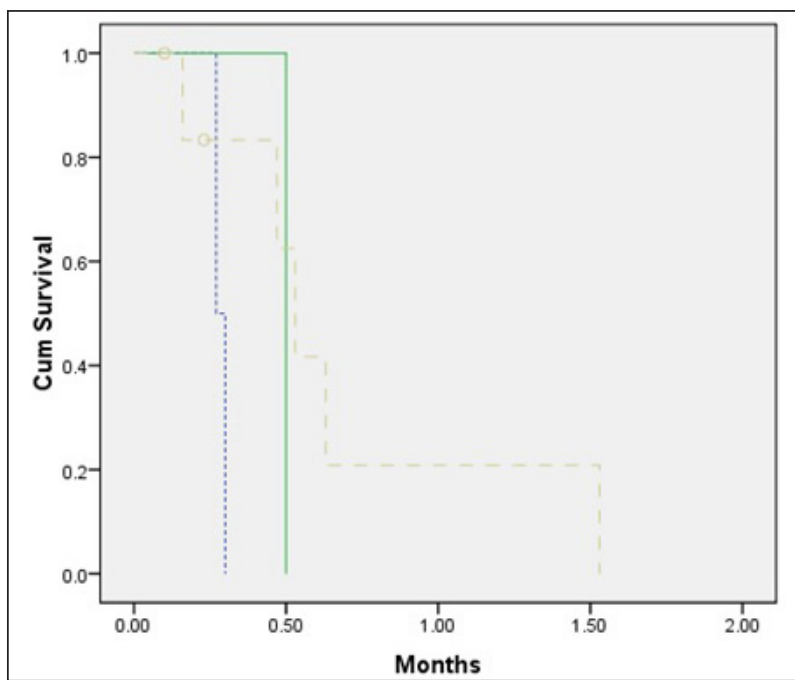

Figure 3. Kaplan-Meier curves showing effect of surgical interventions on overall survival of stage IVC patients 
In our cohort, dyspnea was also a common complaint (68\%) followed by hoarseness (64\%). Lateral neck node (levels II-IV) involvement was observed in $60 \%$ of patients. Involvement of the central nodal basin was more difficult to determine due to confluence with the anterior neck mass.

Distant metastasis was most commonly found in the lungs which presented as multiple nodules/masses ("cannonball" lesions) readily apparent on chest radiography or computed tomography. Pulmonary metastasis contributed to prolonged mechanical ventilation and significant respiratory difficulty, even post-tracheostomy. Bony and hepatic metastasis was also apparent in $30 \%$ and $10 \%$ of patients, respectively.

There is little debate that ATC confined within the thyroid gland (Stage IV-A) is best treated with total thyroidectomy but unfortunately, none of our patients were staged IV-A on diagnosis. The treatment of IV-B disease is more controversial. Several studies have identified prognostic factors that predict the success of surgical resection: age $<60$ 70 years, tumor within thyroid capsule and absence of leukocytosis. ${ }^{13,14}$ As seen in our cohort, patients rarely fulfilled these prognostic criteria. Half of our cohort presented in the elderly ( $>60$-year-old) population. They had sizeable primaries which grew rapidly, sometimes in as fast as two weeks. These factors underscore the need for surveillance and expeditious workup of any rapidly enlarging neck mass or sudden enlargement of previous goiter.

There is an emerging body of evidence that surgery still confers longer cumulative survival even in patients with negative prognostic factors given that the longest survivors had surgical resection as part of their treatment. ${ }^{15,16}$ In our cohort, tumor resectability was defined as any tumor without 270 degree encasement of the common carotid artery or prevertebral fascia extension. This definition is based on the 2002 American Joint Committee on Cancer definition for a T4b tumor which was the convention for determining resectability. ${ }^{17}$ For patients with IV-B disease, tumor was already deemed unresectable in a third of cases. Despite having theoretically resectable tumors in 7 patients, thyroidectomy was only performed in four patients. Two of them also underwent therapeutic bilateral neck dissection for $\mathrm{N} 1 \mathrm{~b}$ disease. Although there were no serious intraoperative morbidities, postoperative hypocalcemia was a common occurrence.

Patients who underwent resection continue to have survival curves similar to patients treated with palliative tracheostomy or those who did not undergo any surgical intervention. A probable reason for this is the difficulty in achieving R0 or even R1 margins. The National Comprehensive Cancer Network thyroid cancer guidelines even mention that R0/R1 resection is achieved usually incidentally for thyroidectomies for other causes..$^{18}$ The difficulty in achieving clear margins puts patients at risk for early recurrence and distant tumor dissemination. Although external beam radiotherapy is a viable treatment for tumor residuals, its effectivity is still unassessed in our setting as patients rarely initiate treatment either due to preference or limitation of funds. In our cohort, all patients who underwent total thyroidectomy were unable to initiate radiotherapy treatment.

Thyroidectomies were rarely performed among IV-C patients due to their poor health status at presentation and the dismal prognosis. In our series, only one patient with metastatic disease underwent thyroidectomy. Most only consented to palliative airway management with tracheostomy. Like the IV-B subgroup, surgical interventions do not seem to significantly improve overall survival in IV-C patients.

Airway obstruction in ATC can be due to extrinsic compression by tumor, direct laryngotracheal invasion or vocal cord paralysis. Stridor occurs in 20-30\% of patients and the degree of obstruction may worsen during the patient's clinical course i.e. during radiotherapy, upon endotracheal manipulation, or onset of pulmonary infection. ${ }^{19}$

The latest American Thyroid Association 2015 guidelines advised against prophylactic tracheostomy in non-obstructed patients due to the significant reduction in their quality of life. ${ }^{20}$ In our institution, we often do it prophylactically due to the rapidly progressive nature of the disease and concern for lack of airway specialists in their home area. However, it is not a procedure without risk, as tracheostomy can pose unique challenges when done in this population, both in the intraoperative and postoperative settings. In most instances, the size of the tumor precludes easy exposure of the trachea and the airway may be considerably displaced by the mass. Prior computed tomographic evaluation is highly recommended to guide the surgeon as to the route of access. The tumor can be debulked to expose the trachea underneath and we routinely send tissue samples for histopathological confirmation of disease. This procedure is ideally done in the operating room and never at bedside. In this cohort, only one patient succumbed to acute upper airway obstruction when endotracheal intubation and emergent tracheostomy failed to secure a patent airway.

The postoperative course of these tracheostomized patients can also be troubling. In our cohort, $40 \%$ of patients experienced recurrent bouts of mucus plugging refractory to routine nursing care which necessitated multiple referrals to the attending otolaryngologist. The occurrence of this complication is due to the design of the tracheostomy tube we use for these patients. We favor an extended tube with a neck flange that can be adjusted anywhere along the length of the tube (TRACOE ${ }^{\circ}$ vario, REF 451 TRACOE medical GmbH, Nieder-Olm, Germany). This bidirectionality affords the surgeon greater flexibility: proximal extension for anterior neck bulk and distal extension for intraluminal tracheal involvement. The trade-off is the absence of an inner cannula 


\section{ORIGINAL ARTICLES}

that makes the management of tracheal secretions more problematic. Furthermore, peristomal bleeding and direct intraluminal extension of the tumor are frequent occurrences that can contribute to intermittent periods of airway obstruction and hypoxemia. All these factors pose significant risks and raise the question whether routine stomal nursing care can be safely done at home.

Our study has several limitations. The primary outcome of diseasespecific mortality, was only reliably determined in $52 \%$ of patients despite extensive efforts to track patients and determine their status. Better follow-up and patient tracking can lead to more reliable data which may translate to more robust conclusions. Another limitation was that we were only able to ascertain postoperative complications from inpatient records. It is highly probable that patients may have suffered other postoperative complications after discharge such as tube displacement/obstruction. Future research should investigate the prognostic value of other interventions such as chemotherapy and/or radiotherapy rendered to patients who did not undergo any surgery.

Currently, we can conclude that the data at hand is insufficient to affirm that tracheostomy or thyroidectomy significantly alter the survival of similarly staged patients. These findings caution us surgeons to be more pragmatic in offering interventions to these patients. They must be advised about the proven benefits and risks surrounding the procedure. Supportive care without surgery is an acceptable alternative if the patient so desires. Ultimately, it is about enabling patients to make informed decisions regarding their care, especially since current treatment efforts continue to be disappointing and the prognosis continues to be grim.

\section{REFERENCES}

1. Aschebrook-Kilfoy B, Ward MH, Sabra MM, Devesa SS. Thyroid cancer incidence patterns in the United States by histologic type, 1992-2006. Thyroid. 2011 Feb;21(2):125-34. DOI: 10.1089/ thy.2010.0021; PMID: 21186939; PMCID: PMC3025182.

2. Gervasi $R$, Orlando $G$, Lerose, $M A$, Amato $B$, Docimo $G$, Zeppa $P$, et al. Thyroid surgery in geriatric patients: a literature review. BMC Surg. 2012; 12 Suppl 1: S16. DOI: 10.1186/1471-2482-12-S1S16; PMID: 23173919; PMCID: PMC34992693.

3. Are C, Shaha AR. Anaplastic thyroid carcinoma: biology, pathogenesis, prognostic factors, and treatment approaches. Ann Surg Oncol. 2006 Apr;13(4):453-64. DOI: 10.1245/ASO.2006.05.042; PMID: 16474910

4. Neff RL, Farrar WB, Kloos RT, Burman KD. Anaplastic thyroid cancer. Endocrinol Metab Clin North Am. 2008 Jun;37(2);525-38. DOI: 10.1016/j.ecl.2008.02.003: PMID: 18502341

5. Nagaiah G, Hossain A, Mooney CJ, Parmentier J, Remick SC. Anaplastic thyroid cancer: a review of epidemiology, pathogenesis, and treatment. J Oncol. 2011;2011:542358. DOI: 10.1155/2011/542358; PMID: 21772843 PMCID: PMC3136148.

6. HundahI SA, Fleming ID, Fremgen AM, Menck HR. A National Cancer Data Base report on 53,856 cases of thyroid carcinoma treated in the U.S., 1985-1995. Cancer 1998 Dec 15; 83(12):2638-48. DOI: 10.1002/(sici)1097-0142(19981215)83:12<2638::aid-cncr31>3.0.co;2-1; PMID: 9874472.

7. Green LD, Mack L, Pasieka JL. Anaplastic thyroid cancer and primary thyroid lymphoma: review of these rare thyroid malignancies. J Surg Oncol. 2006 Dec 15;94(8):725-36. DOI: 10.1002/ jso.20691; PMID: 17131397

8. Chiacchio S, Lorenzoni A, Boni G, Rubello D, Elisei R, Mariani G. Anaplastic thyroid cancer: prevalence, diagnosis and treatment. Minerva Endocrinol. 2008 Dec;33(4):341-57. PMID: 18923370.

9. Polistena A, Monacelli M, Lucchini R, Triola R, Conti C, Avenia S, et al. The role of surgery in the treatment of thyroid anaplastic carcinoma in the elderly. Int J Surg. 2014; 12 Suppl 2:170-76. DOl: 10.1016/j.jijsu.2014.08.347; PMID: 25167852.

10. Baek SK, Lee MC, Hah JH, Ahn SH, Son YI, Rho YS, et al. Role of surgery in the management of anaplastic thyroid carcinoma: Korean nationwide multicenter study of 329 patients with anaplastic thyroid carcinoma, 2000 to 2012. Head Neck. 2017 Jan:39(1):133-139. PMID: 27534388 DOI: 10.1002/hed.24559.

11. Sunga PAL, Cortez ER, Ampil IDC II. Survival of patients with anaplastic thyroid cancer treated with total thyroidectomy plus radiotherapy, or tracheostomy plus radiotherapy. Philipp J Surg Spec. 1995; 50(4): 149-51.

12. LoTE, Jimeno CA, Paz-Pacheco E. Anaplastic Thyroid Cancer: Experience of the Philippine Genera Hospital. Endocrinol Metab (Seoul). 2015 Jun; 30(2): 195-200. DOI: 10.3803/EnM.2015.30.2.195 PMID: 26194079; PMCID: PMC4508264.

13. Kebebew E, Greenspan FS, Clark OH, Woeber KA, McMillan A. Anaplastic thyroid carcinoma treatment outcome and prognostic factors. Cancer 2005 Apr 1; 103(7): 1330-35. DOI: 10.1002/ cncr.20936; PMID: 15739211.

14. Akaishi J, Sugino K, Kitagawa W, Nagahama M, Kameyama K, Shimizu K, et al. Prognostic factors and treatment outcomes of 100 cases of anaplastic thyroid carcinoma. Thyroid. 2011 Nov;21(11):1183-89. DOI: 10.1089/thy.2010.0332; PMID: 21936674.

15. Junor EJ, Paul J, Reed NS. Anaplastic thyroid carcinoma: ninety-one patients treated by surgery and radiotherapy. Eur J Surg Oncol. 1992 Apr; 18(2): 83-88. PMID: 1582515.

16. Haigh PI, Ituarte PH, Wu HS, Treseler PA, Posner MD, Quivey JM, et al. Completely resected anaplastic thyroid carcinoma combined with adjuvant chemotherapy and irradiation is associated with prolonged survival. Cancer. 2001 Jun 15; 91(12): 2335-42. PMID: 11413523.

17. Greene FL, Page DL, Fleming ID, Fritz AG, Balch CM, Haller DG, Morrow M, editors. American Joint Committee on Cancer (AJCC) Cancer Staging Manual, $6^{\text {th }}$ Ed. New York: Springer-Verlag 2002. p 18

18. National Comprehensive Cancer Network. Thyroid Cancer (Version 1.2019). [Accessed 2019 Sep 6]. Available from: http://www.nccn.org/professionals/physician_gls/pdf/thyroid.pdf.

19. Shaha AR, Ferlito A, Owen RP, Silver CE, Rodrigo JP, Haigentz M Jr, et al. Airway issues in anaplastic thyroid carcinoma. Eur Arch Otorhinolaryngol. 2013 Sep;270(10):2579-83. DOI: 10.1007/s00405013-2556-3; PMID: 23689802

20. Smallridge RC, Ain KB, Asa SL, Bible KC, Brierley JD, Burman KD, et al. American Thyroid Association guidelines for management of patients with anaplastic thyroid cancer. Thyroid. 2012 Nov;22(11):1104-39. DOI: 10.1089/thy.2012.0302; PMID: 23130564. 